\title{
Severe Pulmonary Toxoplasmosis Mimicking Viral Pneumonitis after a Third Allogeneic Stem Cell Transplantation in a Man with Acute Lymphoblastic Leukemia
}

\author{
Masahiko Sumi ${ }^{1}$, Satoru Hata ${ }^{2}$, Keijiro Sato ${ }^{1}$, Yuko Fujikawa ${ }^{1}$, Ikuo Shimizu ${ }^{1}$, \\ Toshimitsu Ueki ${ }^{1}$, Daigo Akahane ${ }^{1}$, Mayumi Ueno ${ }^{1}$, \\ Naoaki Ichikawa ${ }^{1}$ and Hikaru Kobayashi ${ }^{1}$
}

\begin{abstract}
A 22-year-old man with acute lymphoblastic leukemia underwent allogeneic stem cell transplantation (alloSCT) twice, then underwent allo-SCT a third time due to relapse. On day 27, he developed acute respiratory distress, and bilateral interstitial infiltrates were noted on CT images. Despite receiving intensive treatment, the patient died on day 32 from progressive respiratory failure. An autopsy revealed evidence of diffuse alveolar damage caused by the genus Toxoplasma. At present, toxoplasmosis is considered to be a rare infectious complication in Japan. However, the actual incidence of toxoplasmosis may be higher than currently believed due to a lack of suspicion of the diagnosis in patients, difficulty in making a diagnosis and low autopsy rates.
\end{abstract}

Key words: Toxoplasma gondii, pulmonary toxoplasmosis, stem cell transplantation, acute lymphoblastic leukemia

(Intern Med 51: 2943-2947, 2012)

(DOI: 10.2169/internalmedicine.51.7837)

\section{Introduction}

Toxoplasma gondii (T. gondii) is a ubiquitous intracellular protozoan. In immunocompetent individuals, $T$. gondii causes asymptomatic infection or fever and lymphadenopathy. However, in immunocompromised patients, T. gondii may cause fulminant disseminated infection (1). Toxoplasmosis occurring after hematopoietic stem cell transplantation (HSCT) is a rare but often fatal complication (2-8). In HSCT recipients, toxoplasmosis infection is usually the result of reactivation of a latent infection rather than the development of a primary infection. The incidence of toxoplasmosis in HSCT recipients shows marked geographical variation depending on local pet-keeping habits, especially the presence of cats, and food contamination with cysts (1). In countries with high endemicity, such as France, specific antibodies can be detected in $50-80 \%$ of the general adult population $(1,2,9)$. In Japan, specific antibodies can be detected in only $10 \%$ of the population $(4,9)$. Although the incidence of toxoplasmosis after HSCT in Japan is not well established, in the United States (US) and France, the incidence is reported to be $0.3 \%$ and $5 \%$, respectively $(5,9)$. The most frequently involved organs are the central nervous system (CNS), lungs and heart, and making a diagnosis is very difficult, especially in patients with lung toxoplasmosis, because the clinical and radiological manifestations are nonspecific $(2,10-12)$. We herein report a case of fatal pulmonary toxoplasmosis mimicking viral pneumonitis in an HSCT recipient that was diagnosed postmortem.

\section{Case Report}

A 22-year-old man was admitted to our hospital in De-

${ }^{1}$ Department of Hematology, Nagano Red Cross Hospital, Japan and ${ }^{2}$ Department of Pathology, Nagano Red Cross Hospital, Japan Received for publication March 27, 2012; Accepted for publication July 24, 2012 Correspondence to Dr. Masahiko Sumi, sumin@nagano-med.jrc.or.jp 


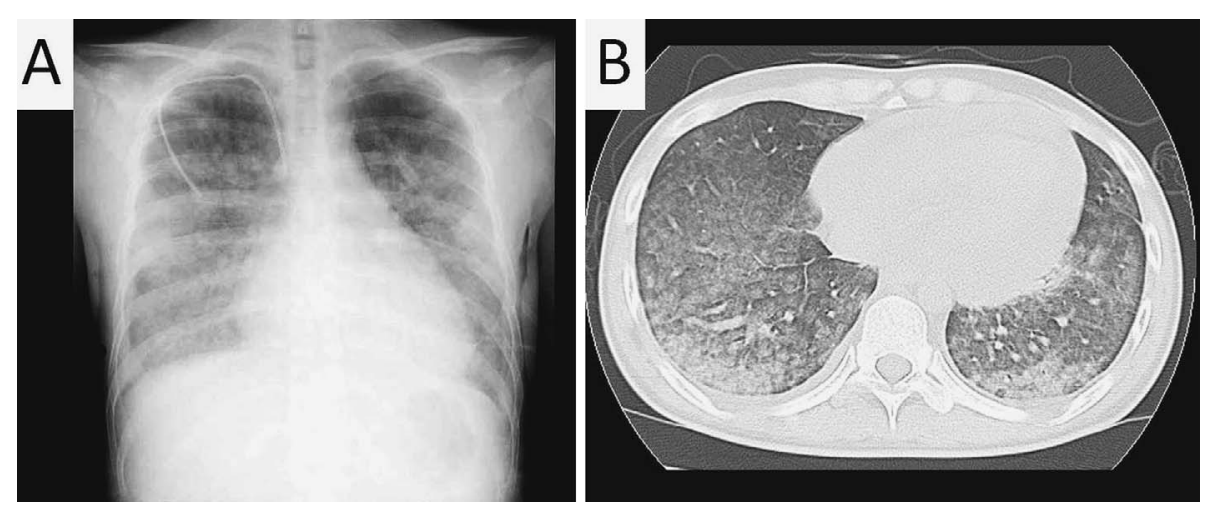

Figure 1. A: Chest X-ray showed bilateral interstitial infiltrates. B: Chest CT images showed ground glass attenuation with coarse nodular opacities.

cember 2005. A diagnosis of precursor B lymphoblastic leukemia was made. The patient had a history of keeping a dog as a pet until one year before admission. He had occasionally eaten raw horsemeat (horsemeat sashimi) prior to admission. Although the patient had achieved hematological complete remission (CR) following the administration of induction chemotherapy, his leukemia relapsed in December 2007. He achieved a second CR following the administration of reinduction chemotherapy, and a first allogeneic stem cell transplantation (allo-SCT) using bone marrow from a human leukocyte antigen (HLA)-matched unrelated donor was performed in May 2008. The conditioning regimen for the first allo-SCT consisted of $60 \mathrm{mg} / \mathrm{kg}$ of cyclophosphamide for two days and six fractionated total body irradiation (TBI) treatments (total: $12 \mathrm{~Gy}$ ). Tacrolimus and short-term methotrexate were used for prophylaxis against graft-versus-host disease (GVHD). The patient's pre-first transplantation serological test was positive for $T$. gondii. The patient developed grade 2 acute skin GVHD and limited-type chronic skin GVHD. However, the leukemia relapsed in January 2009 and a second allo-SCT from HLA 2 antigen-mismatched cord blood was performed in a nonremission state in February 2009. The conditioning regimen for the second allo-SCT consisted of $25 \mathrm{mg} / \mathrm{m}^{2}$ of fludarabine for five days, $40 \mathrm{mg} / \mathrm{m}^{2}$ of melphalan for two days and two fractionated TBI treatments (total: 4 Gy). Tacrolimus was used for GVHD prophylaxis. The patient achieved a third CR and developed grade 2 acute skin GVHD and limited-type chronic skin GVHD. However, another relapse with $36.0 \%$ marrow infiltration by lymphoblasts was confirmed in September 2009. Trimethoprimsulfamethoxazole (T/S) prophylaxis for Pneumocystis jirovecii was administered until relapse. The patient underwent a third allo-SCT with an HLA 1 antigen mismatch from his mother. Serological tests for T. gondii performed at the third transplantation were negative in the patient and positive in the donor. The patient's conditioning regimen consisted of $25 \mathrm{mg} / \mathrm{m}^{2}$ of fludarabine for five days and 90 $\mathrm{mg} / \mathrm{m}^{2}$ of melphalan for two days. Tacrolimus $(0.02 \mathrm{mg} / \mathrm{kg}$, continuous infusion) was used for GVHD prophylaxis. Veno-occlusive disease (VOD) prophylaxis consisted of low- dose heparin and ursodeoxycholic acid. On day 7, a marked fever was observed without evident infection. On day 14, elevated transaminase (alanine transaminase (ALT): 173 IU/ $\mathrm{L}$; aspartate transaminase (AST): $300 \mathrm{IU} / \mathrm{L}$ ) levels, weight gain and thrombocytopenia refractory to platelet transfusion associated with hepatomegaly were observed, and VOD was tentatively diagnosed. At that point, a bone marrow examination revealed severe hypoplastic marrow with $8.3 \%$ macrophages exhibiting hemophagocytosis without evident leukemic blasts. Antimicrobial therapy and supportive therapy, including corticosteroids (prednisolone: $1.4 \mathrm{mg} / \mathrm{kg}$ ), parenteral nutrition, diuretics, low-dose dopamine and albumin recruitment were initiated, resulting in the gradual resolution of VOD; however, the marked fever remained. On day 22, neutrophil engraftment was achieved. However, on day 27 , the patient developed acute respiratory distress, hypoxemia and bilateral interstitial infiltrates on chest X-ray (Fig. 1A). The chest CT images showed ground glass attenuation with coarse nodular opacities and lower lobe distribution in both lungs (Fig. 1B). Because the patient did not have any neurological symptoms, neuroimaging studies, such as brain CT and magnetic resonance imaging, were not performed. A laboratory workup showed the hemoglobin level to be $8.8 \mathrm{~g} / \mathrm{dL}$, the white blood cell count was 2,710 / $\mu \mathrm{L}$ (14\% lymphocytes, $12 \%$ monocytes and $71 \%$ neutrophils) and the platelet count was $34,000 / \mu \mathrm{L}$ with transfusion dependence. The lactic dehydrogenase (LDH), AST and ALT levels were $401 \mathrm{IU} / \mathrm{L}, 66 \mathrm{IU} / \mathrm{L}$ and $118 \mathrm{IU} / \mathrm{L}$, respectively. Tests for $\beta$-D glucan were negative, and coagulation test results were within normal ranges. On the same day, Epstein-Barr virus (EBV: $1.1 \times 10^{4}$ copies $/ 10^{6} \mathrm{WBC}$ ) and cytomegalovirus (CMV: $5.6 \times 10$ copies $/ 10^{6} \mathrm{WBC}$ ) DNA were detected in plasma specimens. Neither lymphadenopathy nor splenomegaly were detected on CT images. Therefore, the patient was tentatively diagnosed with acute pneumonitis associated with EBV or CMV infection. Treatment with ganciclovir, foscarnet and high-dose corticosteroids was initiated and donor lymphocytes were infused on day 31. Despite the administration of aggressive supportive care, the patient died on day 32 due to progressive respiratory failure.

At autopsy, the lungs were found to be congested, and co- 


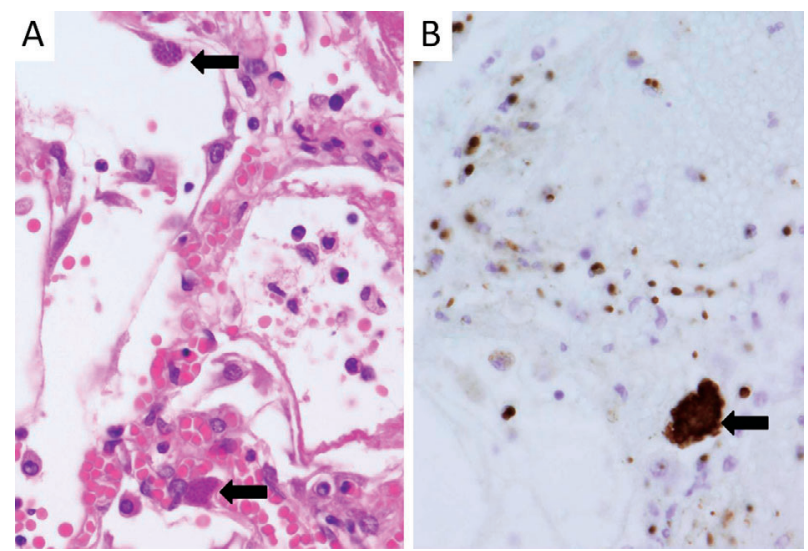

Figure 2. The pathological findings in the lungs at autopsy. Many alveoli contained cells packed with tachyzoites of $T$. gondii (arrows) associated with the development of fibrinous or fibrinopurulent exudates. A: Hematoxylin and Eosin staining (original magnification, $\times 400$ ). B: Immunohistochemical staining of $T$. gondii (original magnification, $\times 400$ ).

agulation necrosis and alveolar fibrinous exudates were observed in both lungs. Many alveoli contained cells packed with tachyzoites of T. gondii (Fig. 2). However, no intracellular inclusions suggestive of herpetic infection were detected in the lungs, and there was no evidence of lymphoproliferative disorders. Immunostaining did not reveal EBV in the lungs. Final pathological diagnoses of pulmonary toxoplasmosis and diffuse alveolar damage were made at autopsy. Although a brain examination was refused at autopsy, there was no evidence of toxoplasmosis in any other organs.

\section{Discussion}

In HSCT recipients, toxoplasmosis usually results from the reactivation of a latent infection rather than the development of a primary infection. However, a few cases of toxoplasmosis occurring in HSCT have been reported in patients with pretransplant serology negative for $T$. gondii, thus suggesting transmission of infection via donor marrow or blood products $(13,14)$. The present patient became seronegative for $T$. gondii after the second allo-SCT due to deterioration of humoral immunity, and the toxoplasmosis probably occurred as a result of reactivation of a latent infection. However, we cannot completely rule out the possibility of a transmission of $T$. gondii via donor marrow because serological tests for $T$. gondii in the donor were positive.

Toxoplasmosis was formerly considered to be a rare infection in recipients of HSCT (1). However, recent singlecenter and multicenter retrospective studies from western countries have suggested that invasive disease may be more common than previously believed (2-6). The incidence varies geographically with the frequency of seropositivity in the general population and ranges from $0.3 \%$ in the US to $5 \%$ in France, where the levels of seropositivity in the population are $3-68 \%$ and $50-80 \%$, respectively $(1,2,9)$. As far as we know, only a few HSCT patients have been reported to have developed toxoplasmosis in Japan. This rarity might be related to the relatively low seropositivity for $T$. gondii in Japan, which has been reported to be approximately $10 \%(4,9)$. Matsuo et al. reported the incidence of toxoplasmosis in Japan among HSCT recipients at four transplantation centers in the Fukuoka Blood Marrow Transplantation Group (4). Two patients who underwent allo-SCT were diagnosed conclusively as having toxoplasmosis encephalitis, which corresponds to a frequency of $0.22 \%$ (two of 925) of all allo-SCT recipients and $2.1 \%$ of seropositive patients.

However, the real incidence of toxoplasmosis is unknown because making an accurate diagnosis is often difficult (15). In the present case, if an autopsy had not been performed, the cause of death would have been considered viral pneumonitis. In countries with a high incidence of toxoplasmosis infection, much progress has been made in the early detection of toxoplasmosis after allo-SCT with serial screening of peripheral blood for $T$. gondii DNA using polymerase chain reaction (PCR). Martino et al. reported the results of a prospective study of the incidence of reactivation of toxoplasmosis in five European transplantation centers. Toxoplasmosis infection was evaluated using PCR for T. gondii DNA in peripheral blood in 106 seropositive adult recipients of HSCT (3). In that report, 16 patients (16\%) had PCR results of peripheral blood specimens that were positive for $T$. gondii. Although all 16 patients with positive PCR results received anti-Toxoplasma therapy after the positive results were revealed, six patients developed Toxoplasma disease, four developed localized encephalitis, one developed pulmonary disease with rapid dissemination and one developed acute disseminated disease. The latter two patients died. In a retrospective study in Japan, it was reported that the incidence of toxoplasmosis among seropositive recipients of allo-SCT is $2.1 \%$ (4), which is lower than that reported in the prospective study by Martino et al. This discrepancy highlights the difficulties of accurately diagnosing toxoplasmosis. The real incidence of toxoplasmosis in Japan may be higher, as lack of suspicion of the disease, difficultly in making a diagnosis and low autopsy rates all contribute to lowering the reported incidence. In Japan, multicenter prospective studies are needed to investigate the real incidence of toxoplasmosis after HSCT.

Mele et al. reported the results of a systematic review of the literature involving 110 cases of toxoplasmosis following allo-SCT (5). Among the patients with disseminated toxoplasmosis, $80 \%$ died with a median survival time of 10 days postdiagnosis and 62.5 days post-allo-SCT. In the study, it was recommended that a diagnosis of disseminated toxoplasmosis should be considered if pulmonary signs and symptoms and/or fever occur in recipients with a risk of reactivation between 30 and 100 days post-allo-SCT (5). However, in cases of disseminated disease, including those with lung or heart involvement, it is very difficult to make an accurate diagnosis and administer appropriate therapy because of the drastic clinical course. In the present case, there 
were only six days between the onset of symptoms and death. Performing PCR for T. gondii in peripheral blood, cerebrospinal fluid and bronchoalveolar lavage fluid is a useful diagnostic tool for detecting toxoplasmosis $(3,7,12$, $14,15)$. However, PCR for $T$. gondii is a time-consuming method because it is not performed routinely in Japan. Laibe et al. reported a case of disseminated toxoplasmosis diagnosed using a Diff-Quick-stained sputum smear analysis (16). Sputum is readily obtainable, even from severely ill patients, and its analysis can be performed immediately. Therefore, careful sputum analysis may allow for accurate diagnosis of pulmonary toxoplasmosis.

Administering prophylaxis or preemptive therapy is a reasonable choice for preventing fatal toxoplasmosis. Two drug combinations have been proven to be efficient in preventing toxoplasmosis in allo-SCT recipients: T/S and pyrimethamine-sulfadoxine (9). In the present case, T/S was administered and toxoplasmosis did not develop after the second allo-SCT. Derouin et al. proposed that prophylaxis for toxoplasmosis should be initiated after 30 days post-alloSCT, as $90 \%$ of cases of toxoplasmosis occur during this time (9). Moreover, they proposed that, if delayed prophylaxis is preferred, weekly follow-up of the recipient using PCR of peripheral blood is recommended for high-risk patients during the entire period without prophylaxis. A study reported by Martino et al. suggested that PCR-based preemptive therapy may prevent death due to toxoplasmosis infection in approximately $80 \%$ of patients who develop Toxoplasma reactivation. In that study, higher PCR density, non-early disease status, second HSCT, cord blood transplantation and a lack of prophylaxis with $\mathrm{T} / \mathrm{S}$ were each found to be associated with an increased incidence of infection according to a univariate analysis (3). Aoun et al. reported the highest risk to be in patients with a haploidentical donor type in their single-center retrospective study (17). The present patient might have had an extremely high risk of reactivation because he was treated three times with allo-SCT, received corticosteroid therapy for hemophagocytosis and could not receive prophylaxis with $\mathrm{T} / \mathrm{S}$ due to liver dysfunction following VOD. In a case like this, preemptive therapy may be a more promising strategy $(3,14)$.

At present, toxoplasmosis is considered to be a rare infectious complication in Japan. However, the incidence of toxoplasmosis may increase because the frequency of performing allo-SCT causing a highly immunosuppressive state (e.g. cord blood or haploidentical stem cell transplantation) is increasing gradually in Japan $(18,19)$. Therefore, physicians should be aware that fatal toxoplasmosis can occur in HSCT patients, and further studies involving a larger number of cases are required to develop strategies to overcome this severe infectious complication. We propose testing all allo-SCT candidates for Toxoplasma IgG positivity to determine who is at risk for reactivation. Seropositive recipients with a high risk of reactivation should receive prophylactic therapy for Pneumocystis jirovecii with T/S instead of pen- tamidine whenever possible. However, several reports have described toxoplasmosis occurring in spite of prophylaxis with T/S. Therefore, routine PCR testing of peripheral blood specimens may be necessary in order to make early diagnoses to avoid fatal toxoplasmosis.

The authors state that they have no Conflict of Interest (COI).

\section{References}

1. Montoya JG, Liesenfeld O. Toxoplasmosis. Lancet 363: 19651976, 2004.

2. Martino R, Maertens J, Bretagne S, et al. Toxoplasmosis after hematopoietic stem cell transplantation. Clin Infect Dis 31: 11881194, 2000.

3. Martino R, Bretagne S, Einsele $\mathrm{H}$, et al. Early detection of Toxoplasma infection by molecular monitoring of Toxoplasma gondii in peripheral blood samples after allogeneic stem cell transplantation. Clin Infect Dis 40: 67-78, 2005.

4. Matsuo Y, Takeishi S, Miyamoto T, et al. Toxoplasmosis encephalitis following severe graft-vs.-host disease after allogeneic hematopoietic stem cell transplantation: $17 \mathrm{yr}$ experience in Fukuoka BMT group. Eur J Haematol 79: 317-321, 2007.

5. Mele A, Paterson PJ, Prentice HG, Leoni P, Kibbler CC. Toxoplasmosis in bone marrow transplantation: a report of two cases and systematic review of the literature. Bone Marrow Transplant 29: 691-698, 2002

6. Busemann C, Ribback S, Zimmermann K, et al. Toxoplasmosis after allogeneic stem cell transplantation: a single centre experience. Ann Hematol 91: 1081-1089, 2012.

7. Mulanovich VE, Ahmed SI, Öztürk T, Khokhar FA, Kontoyiannis DP, de Lima M. Toxoplasmosis in allo-SCT patients: risk factors and outcomes at a transplantation center with a low incidence. Bone Marrow Transplant 46: 273-277, 2011.

8. Meers S, Lagrou K, Theunissen K, et al. Myeloablative conditioning predisposes patients for Toxoplasma gondii reactivation after allogeneic stem cell transplantation. Clin Infect Dis 50: 11271134, 2010.

9. Derouin F, Pelloux H; ESCMID Study Group on Clinical Parasitology. Prevention of toxoplasmosis in transplant patients. Clin Microbiol Infect 14: 1089-1101, 2008.

10. Mariuz P, Bosler EM, Luft BJ. Toxoplasma pneumonia. Semin Respir Infec 12: 40-43, 1997.

11. Saad R, Vincent JF, Cimon B, et al. Pulmonary toxoplasmosis after allogeneic bone marrow transplantation: case report and review. Bone Marrow Transplant 18: 211-212, 1996.

12. Delhaes L, Mraz JC, Fréalle E, et al. Severe pulmonary toxoplasmosis after allo-SCT in two patients: from Toxoplasma genotyping to clinical management. Bone Marrow Transplant 45: 580-583, 2010 .

13. Chandrasekar PH, Momin F; Bone Marrow Transplant Team. Disseminated toxoplasmosis in marrow recipients: a report of three cases and a review of the literature. Bone Marrow Transplant 19: 685-689, 1997.

14. Fricker-Hidalgo H, Bulabois CE, Brenier-Pinchart MP, et al. Diagnosis of toxoplasmosis after allogeneic stem cell transplantation: results of DNA detection and serological techniques. Clin Infect Dis 48: e9-e15, 2009.

15. Cavattoni I, Ayuk F, Zander AR, et al. Diagnosis of Toxoplasma gondii infection after allogeneic stem cell transplant can be difficult and requires intensive scrutiny. Leuk Lymphoma 51: 15301535, 2010.

16. Laibe S, Ranque S, Curtillet C, Faraut F, Dumon H, Franck J. Timely diagnosis of disseminated toxoplasmosis by sputum exami- 
nation. J Clin Microbiol 44: 646-648, 2006.

17. Aoun M, Georgala A, Mboumi K, et al. Changing the outcome of toxoplasmosis in bone marrow transplant recipients. Int J Antimicrob Agents 27: 570-572, 2006.

18. Atsuta Y, Morishima Y, Suzuki R, et al. Comparison of unrelated cord blood transplantation and HLA-mismatched unrelated bone marrow transplantation for adults with leukemia. Biol Blood Marrow Transplant 18: 780-787, 2012.

19. Kurokawa T, Ishiyama K, Ozaki J, et al. Haploidentical hematopoietic stem cell transplantation to adults with hematologic malignancies: analysis of 66 cases at a single Japanese center. Int J Hematol 91: 661-669, 2010.

(C) 2012 The Japanese Society of Internal Medicine http://www.naika.or.jp/imonline/index.html 